\title{
Alimentation en eau en Indonésie
}

\author{
M. Valin \\ SOGREAH Ingénierie
}

L'eau joue un rôle fondamental dans la quasi-totalité des activités humaines. Il n'y a pas de doute que l'alimentation sûre en eau potable est une des fonctions les plus essentielles de l'industrie de l'eau, à côté de la fourniture d'eau pour l'agriculture et l'industrie. Et aujourd'hui le défi devient de plus en plus pressant.

A la fin des années 1980, environ $30 \%$ de la population des régions asiatiques et océaniques n'avait toujours pas accès à une source sûre d'eau potable.

Une des raisons de ce progrès insuffisant est la migration rapide des populations depuis les campagnes vers les agglomérations. Le mauvais entretien et l'inefficacité dans l'utilisation de l'eau sont probablement les problèmes les plus répandus dans ces régions du monde. Par ailleurs, la croissance rapide des agglomérations dans les pays en voie de développement a déjà eu un impact considérable sur la disponibilité d'une alimentation en eau fiable dans les grandes villes.

Cependant, aucune politique de développement de ressources en eau ne peut être efficace sans des incitations économiques qui soient de nature à favoriser les consommations d'eau qui préservent l'environnement. Ceci implique la mobilisation progressive des ressources en eau et le financement d'équipements lourds de distribution d'eau.

L'exploitation de l'industrie d'alimentation en eau est devenue une tâche professionnelle, faisant appel à de grandes compétences. C'est une des raisons pour lesquelles le recours aux entreprises spécialisées du secteur privé s'est aussi largement développé.

Toutefois, comme l'alimentation en eau est une industrie qui requiert des investissements très lourds, à financer sur le long terme, il est juste et raisonnable que la responsabilité dans ce domaine demeure au niveau des gouvernements, afin de parvenir au développement harmonieux de ce secteur au plan national, à la fois dans les domaines techniques et de législation de l'eau.

\section{CONTEXTE}

L'eau est une ressource essentielle, qui répond à de nombreux besoins de la population. Compte tenu de son importance, le droit de l'eau est institué par la Loi n ${ }^{\circ} 11 / 1974$ comme un droit d'usage.

En 1990, le volume total d'eau distribué pour la consommation des ménages et des municipalités en Indonésie s'élevait à environ 13 millions de mètres cubes par jour $\left(150 \mathrm{~m}^{3} / \mathrm{s}\right)$. Près de $70 \%$ de cette eau est consommée par les populations résidant sur l'île de Java. Il est prévu actuellement que le volume d'alimentation en eau s'élèvera à 30 millions de $\mathrm{m}^{3}$ par jour en 2020 (D. Jezeph).
A la fin des années 1940, il y avait des problèmes aigus d'alimentation en eau dans 62 villes d'Indonésie. A l'époque, la capacité cumulée de tous les réseaux d'alimentation en eau était de $390000 \mathrm{~m}^{3}$ par jour. Depuis lors, le gouvernement indonésien a mobilisé des moyens considérables afin d'étendre les réseaux. Cependant, le volume fourni ne dépassait toujours pas $780000 \mathrm{~m}^{3}$ par jour en 1968 (tableau I).

Tableau 1. - Répartition de la capacité des réseaux d'eau potable en Indonésie selon les zones.

\begin{tabular}{|l|c|c|c|}
\hline $\begin{array}{l}\text { Population } \\
\text { en millions }\end{array}$ & 1982 & 1985 & 1990 \\
\hline Urbaine & $33,9(22 \%)$ & $41,2(25 \%)$ & $\begin{array}{r}54,7(30 \%) \\
\text { Semi-urbaine }\end{array}$ \\
$\begin{array}{r}26,2(17 \%) \\
24,3(15 \%)\end{array}$ & $\begin{array}{r}33,9(11 \%) \\
107,6(59 \%)\end{array}$ \\
Rurale & $94(61 \%)$ & $99,4(60 \%)$ & 182,4 \\
\hline Total & 154,1 & 164,9 & 180 \\
\hline
\end{tabular}

Source : Ministère des Travaux Publics.

Au cours de la période récente, le gouvernement a formulé et mis en œuvre une politique nationale pour le développement et l'utilisation rationnelle des ressources et de l'alimentation en eau.

En 1969, le premier plan national quinquennal de développement économique (PELITA I) a été décrété. Les objectifs dans le secteur de l'eau étaient d'augmenter la capacité et de réhabiliter les stations de traitement d'eau potable dans les grandes villes ayant plus d'un million d'habitants (Jakarta, Bandung, Surabaya, Medan et Semarang), et dans 54 villes de taille moyenne. Le volume total d'eau fourni atteignit 1,3 million de $\mathrm{m}^{3}$ par jour $(15000 \mathrm{l} / \mathrm{s})$ en 1973 (tabl. 2).

$\mathrm{Au}$ cours du second plan quinquennal (PELITA II, 1974/1979), l'attention s'est portée sur les aspects qualitatifs et sur la poursuite de la réhabilitation et de l'extension des équipements existants d'alimentation en eau dans les grandes agglomérations, dans les villes moyennes de 100000 à 500000 habitants, et dans les petites villes de 20000 à 100000 habitants. A la fin de ce second plan quinquennal, le volume total fourni avait atteint 2,5 millions de $\mathrm{m}^{3}$ par jour $(29000 \mathrm{l} / \mathrm{s})$.

Bien que PELITA II ait produit des résultats remarquables en termes d'extension des réseaux d'alimentation et de distribution, l'utilisation de plus en plus intensive des 
ALIMENTATION EN EAU EN INDONÉSIE

Tableau 2. - Politique d'alimentation en eau en Indonésie depuis les années 1940

\begin{tabular}{|c|c|c|c|c|}
\hline Pelita & Objectifs & Villes & $\begin{array}{l}\text { Capacité } \\
\text { ( } \mathrm{m}^{3} / \text { jour) }\end{array}$ & Commentaires \\
\hline $\begin{array}{l}\text { Années } \\
1940\end{array}$ & & 62 villes & 390000 & $\begin{array}{l}\text { Extension des réseaux } \\
\text { d'adduction et de distri- } \\
\text { bution }\end{array}$ \\
\hline 1968 & & & 780000 & $\begin{array}{l}\text { Extension des réseaux } \\
\text { d'adduction et de distri- } \\
\text { bution }\end{array}$ \\
\hline Pelita I & & $\begin{array}{l}\text { Djakarta, Bandung, Su- } \\
\text { rabaya, Medan, Sema- } \\
\text { rang et } 54 \text { villes moyen- } \\
\text { nes }\end{array}$ & 1300000 & $\begin{array}{l}\text { Extension et (en 1973) } \\
\text { réhabilitation des usines } \\
\text { de traitement d'eau }\end{array}$ \\
\hline $\begin{array}{l}\text { Pelita II } \\
\text { 1974-1979 }\end{array}$ & & $\begin{array}{l}\text { Métropoles et grandes } \\
\text { villes, villes petites et } \\
\text { moyennes }\end{array}$ & 2500000 & $\begin{array}{l}\text { Extension et réhabilita- } \\
\text { tion des réseaux d'ad- } \\
\text { duction et de distribution }\end{array}$ \\
\hline $\begin{array}{l}\text { Pelita III } \\
\text { 1979-1984 }\end{array}$ & $\begin{array}{l}40 \% \text { de la population } \\
\text { desservie en zones ur- } \\
\text { baines }+32 \% \text { en zones } \\
\text { rurales ; } 17200000 \text { ha- } \\
\text { bitants desservis en eau } \\
\text { par conduite }\end{array}$ & & 3100000 & $\begin{array}{l}\text { Maintien d'une alimenta- } \\
\text { tion équitable en eau trai- } \\
\text { tée aux communautés : } \\
\text { consommation nationale } \\
\text { de base fixée à } 60 \mathrm{l} / \mathrm{hab} / \mathrm{j}\end{array}$ \\
\hline $\begin{array}{l}\text { Pelita IV } \\
\text { 1984-1989 }\end{array}$ & $\begin{array}{l}75 \% \text { desservi en zones } \\
\text { urbaines }+55 \% \text { en zo- } \\
\text { nes rurales ; } 25400000 \\
\text { habitants desservis en } \\
\text { eau par conduite }\end{array}$ & & 4400000 & $\begin{array}{l}\text { Connexions individuelles } \\
\text { portées à } 3000000 \text { en } \\
\text { zones urbaines }\end{array}$ \\
\hline $\begin{array}{l}\text { Opération } d \\
\left.n^{\circ} 14 / 1987\right) \\
\text { Développem }\end{array}$ & $\begin{array}{l}\text { équipements d'alimentatic } \\
\text { te programmes IKK pou }\end{array}$ & $\begin{array}{l}\text { on eau transférée aux e } \\
r \text { les petites villes }<20000\end{array}$ & $\begin{array}{l}\text { htreprises locales } \\
\text { habitants) }\end{array}$ & de production d'eau (PP \\
\hline $\begin{array}{l}\text { Pelita V } \\
1989-1993\end{array}$ & $\begin{array}{l}75 \% \text { desservi en zones } \\
\text { urbaines; } 49000000 \\
\text { habitants desservis en } \\
\text { eau par conduite }\end{array}$ & $\begin{array}{l}710 \text { villes supplémentai- } \\
\text { res desservies : } 660000 \\
\text { connexions supplémen- } \\
\text { taires }\end{array}$ & $\begin{array}{l}\text { capacité sup- } \\
\text { plémentaire de } \\
825000\end{array}$ & $\begin{array}{l}\text { Budget de Rp } 3900 \text { mil- } \\
\text { liards (65\% financé par } \\
\text { la coopération internatio- } \\
\text { nale) }\end{array}$ \\
\hline \multicolumn{5}{|c|}{$\begin{array}{l}\text { Contribution à la Stratégie Nationale de Conservation d'Eau et de Contrôle des Fuites } \\
\text { Pression de la demande industrielle et développement de la gestion des ressources en eau } \\
\text { Implication d'entreprises du secteur privé }\end{array}$} \\
\hline
\end{tabular}

ressources mobilisées et amenées dans les villes est devenue un problème sérieux dans de nombreuses localités, en raison de la croissance économique rapide et de l'urbanisation accélérée. A l'époque, il y avait en outre une insuffisance de main-d'œuvre pour l'exploitation et l'entretien des réseaux.

Pour le troisième plan quinquennal (PELITA III, 19791984), l'objectif fixé consistait à maintenir une alimentation équitable en eau domestique à toutes les communautés par des moyens techniquement et économiquement acceptables. La priorité était accordée aux catégories sociales à faible pouvoir d'achat, aux zones à forte densité de population, aux secteurs isolés où il est difficile d'obtenir de l'eau pure, et aux secteurs souffrant d'épidémies chroniques de maladies transmises par l'eau. PELITA III a réussi la réhabilitation et l'extension des équipements d'alimentation et de distribution d'eau dans les grandes métropoles, dans les villes moyennes et dans les centres desservant les communautés rurales.

L'objectif d'alimentation en eau pure de façon équitable vers toutes les communautés d'Indonésie a amené le Gouvernement indonésien à déterminer le besoin minimum d'eau potable de toute la population à une consommation de 60 litres par personne par jour.

Jusqu'à la fin de PELITA III, la fourniture d'eau urbaine relevait essentiellement de la responsabilité du gouvernement central.

Au cours de PELITA IV (1984-1989), l'alimentation en eau dans les grandes agglomérations a été concédée à des entreprises locales de production d'eau potable sous la 
responsabilité des collectivités locales, conformément à la législation nationale et avec des subventions limitées du gouvernement central. L'objectif de PELITA IV était de faire passer le pourcentage de population des zones rurales desservie par des réseaux de distribution de $40 \%$ à $75 \%$. A cette fin, la réhabilitation et l'extension des équipements existants d'alimentation en eau étaient projetées dans 350 villes, et la réalisation de nouveaux réseaux était projetée dans 1800 des 3350 centres des communautés rurales. Malgré tous les efforts entrepris par le gouvernement, les travaux d'extension ont subi un retard au cours du plan PELITA IV. La capacité de production a néanmoins augmenté de 36000 à $51000 \mathrm{l} / \mathrm{s}$.

Au cours du même plan PELITA IV, des efforts étaient entrepris pour mieux assurer les besoins de consommation d'eau en introduisant un nouveau concept dans les chefslieux des sous-districts ayant une population de moins de 20000 habitants. Ce concept, communément appelé «Programme IKK » (Ibu Kota Kecamatan) est basé sur la fourniture d'un débit continu 24 heures sur 24 . L'écoulement constant est régulé par un dispositif installé sur la borne de connexion de chaque résidence. Le tarif est calculé sur la base d'un forfait mensuel. On a réalisé au cours de cette période plus de 1000 programmes IKK, et malgré quelques cas où les résultats ont été contestés, ce programme a indéniablement permis d'augmenter le service global d'alimentation en eau.

Le plan PELITA V, qui s'est poursuivi jusqu'en $1993 / 1994$, avait pour objectif d'augmenter la proportion de population desservie jusqu'à $75 \%$ de la population totale urbaine et semi-urbaine. Depuis le début de PELITA V, le gouvernement a encouragé les investisseurs privés à développer les réseaux d'alimentation en eau, essentiellement pour les besoins industriels et ceux des grands lotissements résidentiels. On a également invité le secteur privé à collaborer avec les entreprises locales de production d'eau (PDAM) pour la gestion des réseaux sur des bases plus professionnelles. Jusqu'à présent, cette coopération n'a été mise en œuvre que de façon marginale. Cependant, plusieurs projets sont préparés actuellement avec participation du secteur privé.

Un autre problème qui appelle d'urgence la mise en œuvre de solutions appropriées est celui des volumes d'eau non comptabilisés et de la capacité résiduelle de certains réseaux existants.

La pollution des ressources en eau superficielles et souterraines devient également un problème aigu, spécialement dans les zones urbaines, au rythme de l'extension des industries et des zones urbanisées.

Le gouvernement met au point un système de tarification qui aura pour effet de réguler les captages d'eau, afin de préserver l'équilibre des ressources d'eaux souterraines.

\section{POLITIQUE ET STRATÉGIE DE DÉVE- LOPPEMENT}

En développant les programmes d'alimentation en eau, les éléments suivants de la politique globale définie en la matière ont été pris en compte :

- tout développement devrait viser une plus grande efficacité et une plus grande efficience des sytèmes ;

- les capacités des entreprises locales de production d'eau (PDAM) devraient être augmentées ;
- les équipements d'alimentation en eau devraient être considérés comme soutenant directement la croissance économique du pays ;

- l'investissement en systèmes d'alimentation en eau devrait être géré de façon à ce que les réseaux puissent s'autofinancer ;

- les niveaux de consommation passeront de $60 \mathrm{l} / \mathrm{s}$ par jour à des niveaux supérieurs en fonction de la taille des villes, depuis les IKK (moins de 20000 habitants) jusqu'aux métropoles (plus d'un million d'habitants) ;

- l'effort sera porté sur la pleine utilisation de la capacité disponible en étendant les réseaux de distribution d'eau ; - les pertes d'eau devront être minimisées par des efforts spécifiques visant l'amélioration de l'exploitation technique, de l'entretien et de l'administration des réseaux d'alimentation.

\section{STRUCTURES INSTITUTIONNELLES}

Quatre ministères sont impliqués dans l'alimentation en eau en Indonésie :

Le Ministère des Travaux Publics est chargé du développement d'équipements d'alimentation en eau tant pour les populations urbaines que pour les communautés rurales.

Le Ministère de l'Intérieur est chargé de la définition des orientations globales pour l'administration des services de fourniture d'eau dans les provinces.

Le Ministère de la Santé est chargé du suivi et du contrôle de la qualité de tous les équipements d'alimentation en eau.

Le Ministère des Finances et l'Agence Nationale d'Aménagement du Territoire (BAPPENAS) sont les instances responsables de la programmation et de l'allocation des crédits et des aides budgétaires.

Les charges d'exploitation des systèmes de production et d'alimentation en eau sont confiées aux entreprises locales de production et distribution (PDAM), qui sont des sociétés de production financièrement et techniquement autonomes, agissant sous le contrôle des collectivités locales. Il existe 276 PDAM en Indonésie (en 1993). A présent, la capacité totale des PDAM est de $53000 \mathrm{l} / \mathrm{s}$ d'eau traitée distribuée par conduite à 18 millions de personnes. Le nombre total d'employés des PDAM est de 24000.

Les BPAM sont établis pour de nouvelles sociétés transférées aux gouvernements locaux. Les BPAM sont sous la surveillance des branches du Ministère des Travaux Publics qui sont responsables de la réalisation des équipements d'alimentation en eau. Lorsque les BPAM deviennent capables de s'autogérer, ils sont réorganisés en BPAM indépendants. Il y avait 17 BPAM en Indonésie en mars 1993, fournissant de l'eau traitée par conduite à $3 \%$ de la population urbanisée.

L'Association de Distributeurs d'Eau d'Indonésie (Perpamsi), un organisme semi-officiel fondé en 1972, est une association de sociétés locales de distribution d'eau, qui a pour objectif de rassembler tous les éléments nécessaires pour le développement de réseaux d'alimentation en eau. Perpamsi organise des programmes de formation et d'enseignement et des ateliers en coordination avec les PDAM, et réalise des programmes de recherche et des études en collaboration avec des organismes internationaux. Elle 
mène par ailleurs des campagnes de sensibilisation du public dans les domaines de la consommation d'eau et de l'environnement.

\section{AIDE ÉTRANGÈRE}

La situation internationale changeant très rapidement, des besoins nouveaux et pressants pour le financement d'aménagements apparaissent dans les pays en voie de développement, puisque ces pays sont toujours confrontés à des difficultés économiques et ont toujours besoin d'aides étrangères. Il est ainsi nécessaire de répondre aux besoins de plus en plus diversifiés de ces pays, y compris ceux qui résultent de nouvelles problématiques mondiales.

En revanche, puisqu'il y a des limites aux possibilités de financement par les pays bailleurs de fonds, il est essentiel d'utiliser rationnellement les ressources, ce qui implique la coopération et la coordination avec les principaux organismes de financement internationaux, tels que la Banque Mondiale, la Banque Asiatique de Développement, etc...

De plus, pour que l'assistance aux pays en voie de développement soit menée efficacement, des mesures ont été instituées pour promouvoir des liens permanents de coordination avec les organismes de financement bilatéraux et multilatéraux, les études de politiques de développement, l'assistance au développement et les études d'identification et de définition de projets.

Des études de post-évaluation cherchent par ailleurs à refléter les leçons à tirer de l'assistance au développement et des projets et programmes spécifiques.

\section{VםRÔLE DU SECTEUR PRIVÉ DANS L'ALIMENTATION EN EAU}

Beaucoup de raisons sont avancées pour expliquer pourquoi l'industrie de l'eau s'oriente vers la privatisation. Un des principaux facteurs cités est le décalage entre les besoins pressants de financement par les gouvernements centraux et les crédits disponibles pour l'investissement, pour que le secteur de l'eau réponde aux exigences des consommateurs et des communautés visant des normes de service améliorées.

La privatisation offre une solution par la mobilisation de fonds privés permettant le financement de programmes d'investissement nécessaires pour atteindre l'objectif de normes de service. Parmi les enjeux, il y a aussi l'acceptation par les consommateurs de ces nouvelles normes de service, et leur accord sur les tarifs plus élevés qui en résultent.

Tout ceci présuppose une volonté politique préalable en faveur de la privatisation des services de distribution d'eau. Cependant, les contraintes économiques et financières poussent beaucoup de ces pays à se tourner vers les sociétés étrangères pour participer à des dispositifs de financement en capital ou par concessions, mobilisant des fonds locaux, offshore ou éventuellement provenant de fonds d'assistance.

\section{COOPÉRATION BILATÉRALE FRAN- ÇAISE DANS LES SECTEURS DE L'EAU ET DE L'ENVIRONNEMENT}

Compte tenu de la rapide croissance économique du pays, le Gouvernement d'Indonésie souhaite mettre en place des structures nationales et une législation appropriées, se prêtant à la mise en cuvre d'une politique nationale dans le secteur de l'eau. Le Gouvernement français a institué en la matière une coopération bilatérale offrant une assistance technique et financière au gouvernement indonésien.

Plus particulièrement, et depuis plusieurs années déjà, le gouvernement indonésien a cherché à appliquer la gestion intégrée des ressources en eau à travers une approche par bassin versant, en vue de développer chaque bassin d'une manière intégrée et complète. A cette fin, 90 principaux bassins versants ont été identifiés. On envisage ainsi la gestion intégrée de l'agriculture irriguée, du développement industriel, de l'hydroélectricité, de l'alimentation en eau potable, de la pêche, des loisirs sur l'eau, de la pollution et de la protection contre les inondations. D'autres aspects pris en compte également sont la lutte contre les érosions, les forêts, la construction de barrages-réservoirs et la conservation globale des ressources en sols cultivables et en eau.

Ce nouveau concept de gestion intégrée des bassins versants s'est renforcé depuis que le gouvernement indonésien a établi (par décret présidentiel n ${ }^{\circ} 5$ daté du 12 février 1990) un nouvel organisme public appelé « Perum Jasa Tirta ", un établissement public de distribution d'eau qui gèrera les ressources du bassin de Brantas dans l'Est du Java.

L'idée essentielle est d'appréhender les problèmes de l'eau dans le cadre de leurs limites naturelles, en ayant le souci de prendre en compte les autorités existantes. L'expérience française de la gestion des bassins versants depuis une vingtaine d'années a montré que c'est un système efficace. Il y a par ailleurs quelques similitudes entre les structures administratives des deux pays. Dans le système français, le principe consiste à faire payer aux consommateurs d'eau les volumes consommés et la pollution correspondante. Les sommes collectées sont ensuite consacrées aux mesures contre la pollution et à la réalisation de nouveaux projets d'adduction et d'extension des réseaux.

Il est utile de mentionner enfin l'effort particulier de programmation d'actions d'enseignement et de formation en France d'ingénieurs indonésiens, en coopération avec le Ministère des Travaux Publics, dans le domaine des routes, de l'infrastructure d'eau et du développement de ressources en eau.

\section{VII — L'EXEMPLE DE DJAKARTA}

Djakarta est une province d'Indonésie qui a un statut administratif particulier en tant que capitale, comme précisé par la loi n 11/1990.

DKI Djakarta a une surface de $660 \mathrm{~km}^{2}$, comprenant les îles de Pulau Seribu. La ville de Djakarta avait une population totale de 7,35 millions d'habitants en 1984. Il est prévu que la population atteigne 10 millions en 1995, avec une densité d'environ 10000 habitants par kilomètre carré, et quelque 12 millions d'habitants en 2005. 
Le développement de la ville depuis quelques années a transformé l'image de Djakarta. La croissance économique moyenne a atteint près de $7 \%$ par an au cours de la période 1984-1989. En 1989 la croissance a été de $9 \%$.

La population actuellement desservie représente $30 \%$ environ de la population totale des zones de desserte. Cependant, les conditions de distribution d'eau potable sont loin d'être satisfaisantes. PAM Jaya parvenait à une production totale de $12500 \mathrm{l} / \mathrm{s}$ en 1993. La production sera augmentée à $18000 \mathrm{l} / \mathrm{s}$ après la réalisation des stations de traitement d'eau de Buaran et de Cisadane.

La longueur des conduites principales était de $27000 \mathrm{~m}$ dans les années 1920 , de $79000 \mathrm{~m}$ dans les années 1950 et de $145000 \mathrm{~m}$ dans les années 1970 . A la fin des années 1980 , la longueur du réseau de conduites était de $250000 \mathrm{~m}$.

En 1984-1985 un nouveau schéma directeur a été préparé pour la période 1985-2005. Divisé en deux phases distinctes de 10 ans, 1985-1995 et 1995-2005, ce schéma directeur visait à définir les priorités dans la production et la distribution d'eau et pour la réhabilitation et l'extension du réseau existant de conduites dans la région DKI Djakarta. Le schéma directeur a défini six zones de distribution à mettre en œuvre sous le programme PJSIP Phase 1 .
Après la préparation du schéma directeur, deux études de faisabilité ont été entreprises pour deux nouveaux systèmes :

- Buaran I et II par des consultants japonais pour l'Est de Djakarta $\left(5 \mathrm{~m}^{3} / \mathrm{s}\right)$,

- Cisadane I par Sogreah pour l'Ouest de Djakarta $\left(3 \mathrm{~m}^{3} / \mathrm{s}\right)$.

Sogreah a participé en 1989-1990 et en association avec d'autres consultants français aux prestations de conseil pour la "soudure ", destinées à permettre l'utilisation immédiate des fonds mobilisés sous l'accord entre le gouvernement indonésien et la Banque Mondiale, pour le projet d'amélioration du système PAM Jaya (PAM Jaya System Improvement Project, ou PJSIP), sans attendre le délai nécessaire à la préparation du projet dans sa totalité.

Par ailleurs, sous la coordination du Ministère des Travaux Publics, Cipta Karya, Sogreah a participé à l'étude et à la maîtrise d'œuvre des travaux du projet de Cisadane Phase I, comprenant entre autres aménagements, l'usine de traitement d'eau de Serpong, pour laquelle une convention préalable de prêt avait été conclue entre les gouvernements indonésien et français.

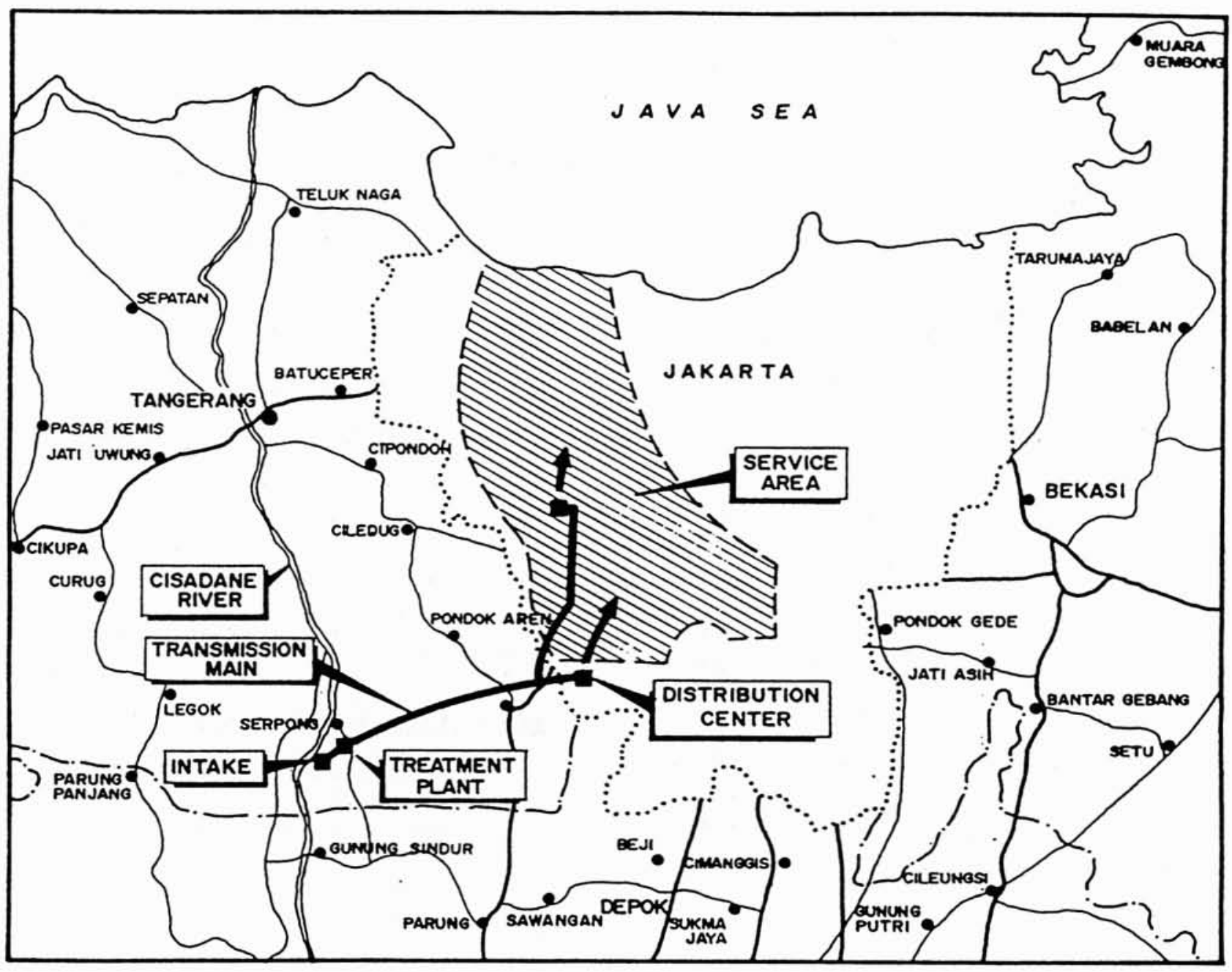

Situation de Jakarta. 
Le projet de Cisadane défini par le schéma directeur d'alimentation en eau de Djakarta de 1985 visait à acheminer $260000 \mathrm{~m}^{3}$ par jour à l'Ouest de Djakarta, pour desservir une population d'environ 1500000 habitants dans les zones PJISP 4 et 5. L'usine de traitement d'eau et la station de pompage associée, pour lesquelles l'équipement a été fourni par Degrémont, ont été mises en service en 1992.

Les réseaux des zones PJISP 4 et 5, étudiés par SOGREAH et actuellement en cours de réalisation sous la surveillance conjointe de PAM Jaya et de SOGREAH, font partie du programme de PAM Jaya pour l'amélioration et l'extension des moyens de production et de distribution, programme cofinancé par le gouvernement indonésien et la Banque Mondiale sur la période 1990-1996.

Les études pour l'ensemble de ces projets ont été réalisées en étroite collaboration avec des bureaux d'ingénieurs conseils indonésiens.

Le projet d'adduction de Cisadane I et le projet d'équipement des zones PJISP 4 et 5 comportent les éléments suivants :

- Situés à Serpong, à 15 kilomètres à l'Ouest de Djakarta sous l'autorité de PDAM Tangerang, les équipements de production comportent une prise d'eau associée à une station de pompage d'eau brute prélevant $3,2 \mathrm{~m}^{3} / \mathrm{s}$ dans la rivière Cisadane, une usine de traitement d'eau avec une capacité de production de $3 \mathrm{~m}^{3} / \mathrm{s}$, et une station de pompage d'eau traitée.

- La conduite d'adduction principale (diamètre $1500 \mathrm{~mm}$, longueur $11,4 \mathrm{~km}$ ) amènera l'eau traitée aux centres de distribution R4 et R5 situés respectivement à l'Ouest et au Sud-Ouest de Djakarta, à Kebon Jeruk et à Lebak Bulus.

- Chacun des deux centres de distribution R4 et R5 comporte deux réservoirs de service de $22500 \mathrm{~m}^{3}$ de capacité totale et une station de pompage qui alimente le réseau directement à travers des groupes variables ou à vitesse constante, le débit d'équipement étant de $455 \mathrm{l} / \mathrm{s}$. Avec les groupes à vitesse variable, le système de contrôle permettra de stabiliser la pression de service en fonction de la demande.

- Le réseau de conduites principales des zones R4 et R5 s'étend sur $62 \mathrm{~km}$, et comporte des conduites en acier soudées, dont le diamètre varie entre 300 et $1200 \mathrm{~mm}$.

- Les $650 \mathrm{~km}$ de conduites secondaires et tertiaires du réseau de distribution sont essentiellement constitués de conduites en PVC dont le diamètre varie entre 50 et $250 \mathrm{~mm}$.

Concernant les principes d'exploitation et la télétransmission, il est clair que les données essentielles devront être à la disposition des exploitants dans les lieux stratégiques répartis sur le réseau, c'est-à-dire à l'usine de traitement de Serpong et aux centres de distribution à Kebon Jeruk et à Lebak Bulus. Les données transmises comprendront les variables hydrauliques telles que débits, niveaux et pressions. Les données seront transmises par une ligne pilote en fibre optique placée le long de la conduite principale d'adduction d'eau.

\section{VIII $\square$ CONCLUSION}

L'eau est vitale pour tout le monde, et pour toute activité humaine, qu'elle soit économique, sociale ou environnementale. Dans tous les pays, l'utilisation et la gestion de l'eau sont profondément enracinées dans le contexte global historique, culturel et géographique.

Alors que l'Indonésie s'apprête à entrer dans le $\mathrm{XXI}^{\mathrm{c}}$ siècle, le gouvernement a mis en œuvre une politique et une stratégie de développement dans le secteur de l'eau. La deuxième période de planification à long terme sur 25 ans (PJPT II) devrait voir une croissance annuelle de $5,25 \%$. Les estimations actuelles laissent supposer que la population de l'Indonésie pourrait atteindre 280 millions d'habitants en 2020. Il est prévu également que les fonds d'investissement proviendront, soit du Gouvernement, soit du secteur privé, tandis que la maîtrise d'œuvre sera confiée pour l'essentiel au secteur privé.

Les changements qui ont eu lieu progressivement au cours de la période PJPT I se poursuivront à un rythme bien plus rapide.

Concernant l'alimentation en eau urbaine, les systèmes de distribution et les fournitures d'eau en volume, la synchronisation effective de la programmation et de la réalisation apportera directement des avantages à la fois aux usagers domestiques et aux consommateurs industriels.

L'Indonésie peut tirer parti de l'expérience acquise par les autres pays et adapter les modes d'application aux besoins spécifiques nationaux. Etape par étape, l'Indonésie créera son propre système fondé sur ses valeurs et sur ses ressources humaines. 